Anales de Geografía de la Universidad Complutense ISSN: 0211-9803

http://dx.doi.org/10.5209/AGUC. 60472

\title{
Valoración y clasificación de la irregularidad interanual: Aplicación en la cuenca del Ebro
}

\author{
Miguel Sánchez Fabre ${ }^{1}$ \\ Recibido: 2 de junio del 2016 / Enviado a evaluar: 28 de julio del 2016 / Aceptado: 14 de diciembre del 2017
}

Resumen. La irregularidad interanual es una de las principales características que definen el comportamiento de los ríos. Su magnitud debe ser tenida en cuenta en la gestión de los caudales de los cursos fluviales. Es, por tanto, conveniente establecer una clasificación de esta irregularidad interanual de los ríos, a través de una mecánica simple. El objetivo de este trabajo es elaborar una propuesta de categorización de la irregularidad interanual en clases o tipos bien definidos y contrastados. Previamente hay que determinar un método sencillo y riguroso para obtener los valores de irregularidad interanual, a partir de las series de datos de aforos. La aplicación de esta propuesta en los ríos de la Cuenca del Ebro permite establecer la presencia de 5 niveles o categorías de irregularidad interanual, con una distribución marcada por diferentes factores geográficos. En ella se aprecia un marcado contraste entre los ríos de las márgenes izquierda y derecha del Ebro.

Palabras clave: Ríos; irregularidad interanual; régimen fluvial; cuenca del Ebro.

\section{[en] Assessment and classification of the interannual variability: Application in the Ebro basin}

Abstract. The interannual variation of the water discharge is one of the main characteristics that defines the behavior of rivers. The magnitude of this irregularity must be taken into account in managing the flow of watercourses. Therefore, it is desirable to establish a classification of this interannual variation of rivers, by using simple procedures. The aim of this work is to develope a proposal of categorization of the interannual variation in well defined and contrasted types. First of all, it is necessary to determine a simple and accurate method to obtain interannual irregularity values, from the series of traffic counts data. Thanks to the implementation of this proposal in the Ebro's Basin rivers, it is possible to distinguish between 5 levels or categories of the interannual variation, with a geographical distribution marked by different factors. This shows a strong contrast between the rivers on the left and right banks of the Ebro.

Keywords: River; interannual variability; river regimes; Ebro basin.

1 Departamento de Geografí y Ordenación del Territorio. Universidad de Zaragoza.

E-mail: msanchez@unizar.es 


\section{[fr] Évaluation et classification de l'irrégularité interannuelle: Application dans le basin hydrographique de l'Ebro}

Résumé. L'irrégularité interannuelle est l'une des principales caractéristiques qui définissent le comportement des rivières. L'ampleur de cette irrégularité exige une attention particulière dans la gestion du flux des cours d'eau. Il est donc souhaitable d'établir une classification de cette irrégularité interannuelle des cours d'eau, à travers une mécanique simple. L'objectif de ce travail est d'élaborer une proposition de catégorisation d'irrégularité interannuelle dans des classes ou des types définis et vérifiés. Une méthode simple et rigoureuse pour obtenir les valeurs d'irrégularité interannuelle doit être définie au préalable, à partir des séries de données hydrométriques. L'application de cette proposition dans les rivières du bassin de l'Èbre permet d'établir la présence de 5 niveaux ou catégories d'irrégularités, dont la répartition est marquée par différents facteurs géographiques. Cela montre un fort contraste entre les rivières situées sur les rives gauche et droite de l'Ëbre.

Mots clés: Rivière; irrégularité interannuelle; régime fluvial; bassin hydrographique de l'Ebre.

Cómo citar. Sánchez Fabre, M. (2018): Valoración y clasificación de la irregularidad interanual: Aplicación en la cuenca del Ebro. Anales de Geografia de la Universidad Complutense, 38(1), 137-160.

Sumario. 1. Introducción. 2. Metodología. 3. Resultados y discusión. 3.1. Determinación de la irregularidad: Valores de coeficiente de caudal y desviación típica. 3.2. Propuesta de clasificación de la irregularidad interanual. 3.3. La irregularidad interanual en la cuenca del Ebro. 4. Conclusiones. 5. Bibliografía.

\section{Introducción}

Cuando a mitad del siglo XX Masachs (1948) publicó su conocido trabajo "El régimen de los ríos peninsulares", dedicó un importante apartado a su irregularidad interanual. Señaló que los valores medios de caudal para un periodo prolongado de tiempo "oscilan alrededor de aquellos y sufren una variación temporal cuyos límites extremos, definidos en una serie muy larga de observaciones, caracterizan la irregularidad". Y añade que la irregularidad de un río, en una estación de aforo, se expresa "por medio del coeficiente de irregularidad obtenido dividiendo el caudal anual máximo del periodo observado por el caudal anual mínimo del mismo". Dicho esto, apunta que todavía el periodo de observaciones existente no es lo suficientemente dilatado para que los valores que el obtiene no vayan a variar en el futuro.

A partir de entonces, la mayoría de los trabajos entre cuyos objetivos está la caracterización del comportamiento hidrológico de un río o una cuenca hidrográfica han analizado la irregularidad interanual, haciendo algunos de ellos referencia al coeficiente de irregularidad. En un ámbito próximo sirven de referencia los trabajos sobre el Ebro de Davy (1975) y Ollero (1996), sobre los ríos pirenaicos de García Ruiz et al. 1985 y 2001, sobre ríos mediterráneos de Gil Olcina (1968 y 1988), Conesa (1985), Conesa y Martínez-Alcocer (1995), Triviño y Ortiz (2004), Iglesias et al. (2005), Morales et al. (2005), Martín (2010) y Conesa y Pérez (2014). Pero esta 
consideración de la irregularidad interanual se extiende a cualquier ámbito territorial. Sirven de ejemplos Robertson y Mechoso (1998), en los ríos Negro y Uruguay, Restrepo y Kjerfve (2000) en el río Magdalena, ó Wang et al. (2006), en el río Amarillo.

Figura 1. Localización del área de estudio.

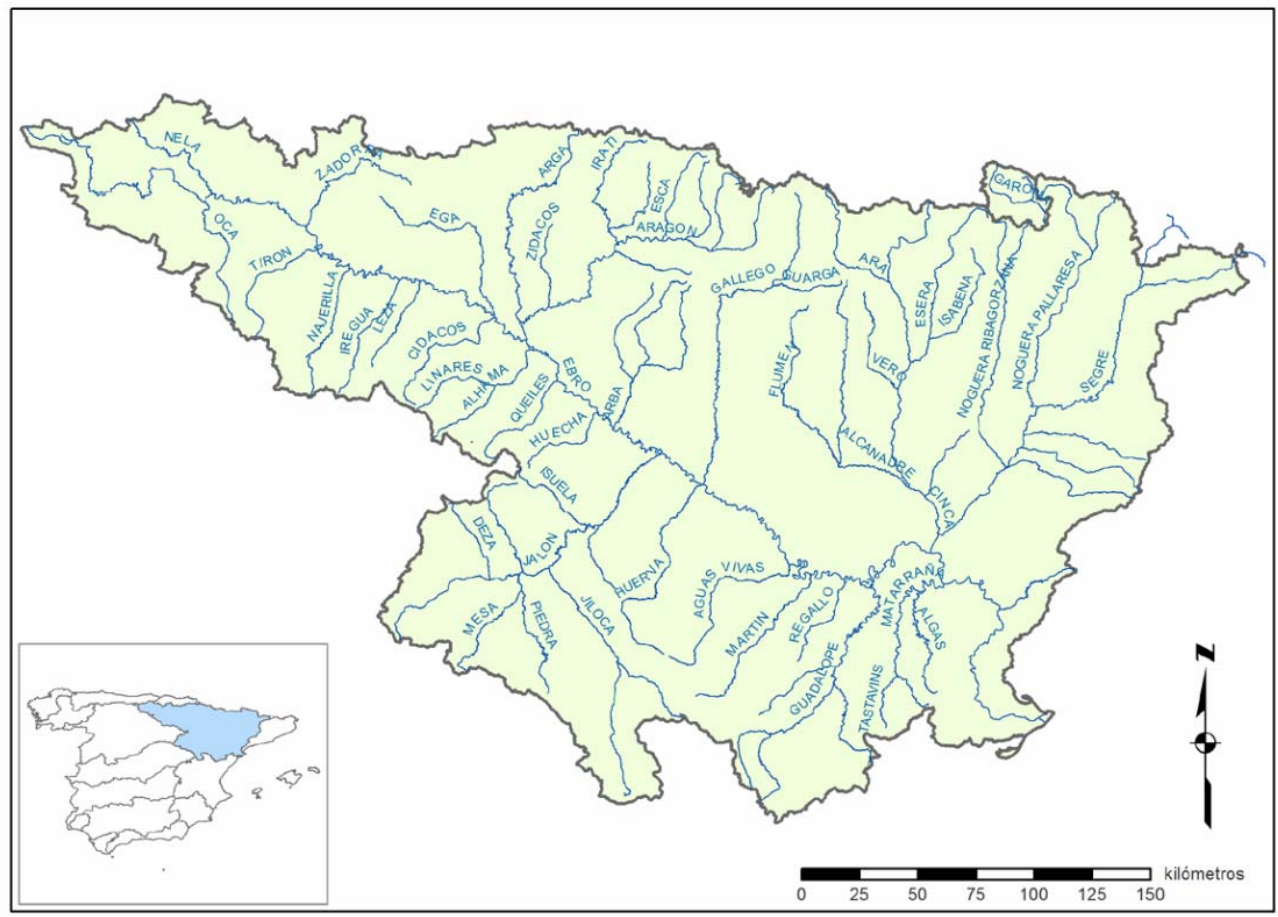

Fuente: Confederación Hidrográfica del Ebro. Elaboración propia.

En varios de los trabajos citados se hace referencia a la importancia que la irregularidad interanual tiene en la gestión de los recursos hídricos. Esta idea cobra especial relevancia en diferentes trabajos o informes realizados por o para organismos responsables de gestión hidraúlica (Gobierno Vasco, 2003; Iberinsa, 2005; Agencia Catalana del Agua, 2015). Así, por ejemplo, puede condicionar la regulación de los embalses o la producción de energía hidroeléctrica. Pero la irregularidad interanual también es relevante en el comportamiento de los ríos y la evolución de los sistemas fluviales, ya que acentúa el rigor y los efectos de estiajes y crecidas, que si son muy frecuentes y de grandes proporciones aumentan a su vez la irregularidad, determina la conservación y estado de diferentes ecosistemas fluviales, o influye en la dinámica 
hidrogeomorfológica de los cursos fluviales tanto de caudal permanente como discontinuo.

Esta importancia, en la doble vertiente de caracterizar el comportamiento fluvial y de marcar la gestión de recursos hídricos, nos hace considerar interesante elaborar una propuesta de clasificación de diferentes niveles o grados de irregularidad. Cada uno de ellos marca un ritmo interanual diferente en la circulación de agua por los ríos y, por tanto, una variación importante en las aportaciones disponibles y en la manera más adecuada de gestionarlas.

Por ello, el objetivo principal de este trabajo es elaborar una clasificación con diferentes niveles de irregularidad interanual. Esta clasificación permitirá asignar a cada río, en cada estación de aforo, un tipo de irregularidad. Como objetivos específicos determinar los criterios para establecer la clasificación y, además, aplicarla a un territorio, la cuenca del Ebro, para testar su aplicabilidad y su ajuste (bondad).

Dicha cuenca del Ebro es, por tanto, el ámbito territorial del presente trabajo (Fig. 1). Sus $85.001 \mathrm{~km}^{2}$ la convierten en una de las más extensas de la Península Ibérica. En Tortosa, muy cerca de su desembocadura, la aportación media anual asciende hasta 13.448,9 $\mathrm{Hm}^{3}$. Aproximadamente tres cuartas partes de este volumen de agua llega hasta el Ebro, curso principal de la cuenca, desde sus afluentes de la margen izquierda, muchos de ellos con cabecera en los Pirineos, en tanto que la mayor parte de los tributarios por su margen derecha son poco caudalosos.

\section{Metodología}

El punto de partida del trabajo es la descarga de datos desde el Sistema del Anuario de Aforos (http://sig.magrama.es/aforos). Concretamente los correspondiente a las aportaciones anuales de aforos de la Cuenca del Ebro. Inicialmente se planteó considerar 143 estaciones de aforo, aunque el análisis de los registros redujo este número hasta 119 aforos, bien porque las series no eran suficientemente largas o bien porque faltara un porcentaje de registros significativo. Se han usado los datos del caudal que pasa por las estaciones de aforo, sin naturalizar, ya que estas cantidades de agua son las que marcan la irregularidad real en la circulación del agua por los cauces $\mathrm{y}$, por tanto, las que interactúan con los ecosistemas fluviales y actúan como agentes de los procesos hidromorfológicos.

A la vista de la información disponible, se planteó determinar la irregularidad interanual de cada aforo a partir de la serie de datos registrados entre 1950/1951 y 2009/2010, lo que supone un periodo homogéneo y extenso (60 años). Las estaciones que ofrecen el dato de aportación anual para este periodo son 82. Aunque para establecer la irregularidad interanual es mejor cuanto más prolongado sea el periodo con valores, para complementar la información extraída de estos 82 aforos se han añadido otros 37 (Anexo 1) con continuidad en sus datos entre 1975/1976 y 2009/2010 (35 años). Conviene señalar que el conjunto de series consideradas está completo en casi un $90 \%$ de los registros. 
Con los valores de aportación anual de cada aforo se ha calculado el coeficiente de irregularidad, del modo que lo planteó Masachs (1948) y lo han seguido haciendo muchos autores (García Ruiz et al., 1985 y 2001; Conesa y Martínez-Alcocer, 1995; Triviño y Ortiz, 2004; Martín, 2010). Este coeficiente pone en relación el máximo registro de aportación anual de la serie, con el mínimo. Así, nos muestra el número de veces que la aportación más elevada multiplica a la más baja. Sus resultados pueden oscilar desde aproximadamente 2 hasta casi el infinito.

Sin duda, los valores de este coeficiente de caudal están muy condicionados por la extensión temporal de la serie y por la magnitud de las aportaciones fluviales, pareciendo más fácil obtener valores elevados en ríos poco caudalosos. En este trabajo el condicionante de la duración de las series está minimizado al disponerse de un buen número de aforos con periodo de recogida de información muy largo. La prolongada duración de las series permite considerar como bastantes estables los valores de coeficiente de irregularidad obtenidos, aunque siempre expuestos a una aportación anual excepcional, de forma aislada en alguno de los aforos. También permite estimar como característicos de cada aforo los valores de dispersión calculados.

El condicionante de la abundancia del caudal se ha intentado paliar llevando todos los valores a la misma magnitud. Es decir, se ha considerado el módulo anual de la serie para cada aforo, atribuyéndole el valor 1 . Después, se han convertido los valores de aportación anual a su proporción respecto a ese valor unitario del módulo, simplemente dividiendo cada registro de aportación anual por dicho módulo (razón). Así, independientemente de la abundancia de cada río, todos se miden en igual orden de magnitud. Además, de este modo se expresa mejor la proporcionalidad de las variaciones que cada año sufre la disponibilidad de caudal en los ríos.

A partir de los valores obtenidos con la mencionada razón numérica se ha determinado la desviación estándar o típica, con la intención de observar cómo se disponen los datos en torno a la media (módulo) en cada aforo. Consideramos que este dato es muy relevante para determinar la irregularidad interanual, ya que matiza el coeficiente de irregularidad. Este último puede ser elevado con sólo dos años de una larga serie con aportación muy distinta. Sin embargo, la desviación estándar lo que nos va a indicar es si son muchos o pocos los casos de años con aportaciones muy elevadas o muy bajas, hecho que define la irregularidad tanto como un alto coeficiente.

También se ha calculado, a partir de los datos de aportaciones anuales, el coeficiente de variación por si sus resultados resultaban más expresivos que la desviación estándar obtenida según se ha explicado. En los 119 aforos considerados el resultado de este coeficiente de variación ha sido similar al de la desviación típica. Por ello, el uso como referente de la tipificación de uno u otro de estos cálculos resulta completamente indiferente.

A partir de la combinación de los resultados del coeficiente de irregularidad y de la desviación típica, se han determinado varios grupos o niveles de irregularidad. Se parte de un coeficiente de irregularidad más o menos elevado, pero se tiene también 
en cuenta la dispersión que las aportaciones de los diferentes años de la serie poseen respecto a la media (módulo).

Finalmente, se asigna categoría a cada uno de los aforos considerados en los ríos de la cuenca del Ebro. Esta clasificación, así como su distribución espacial, se plasman en la cartografía elaborada con ArcGIS. Las bases cartográficas de la red fluvial, divisoria de la cuenca y estaciones de aforo han sido descargadas del SITEbro. En la base de datos de las estaciones de aforo se ha incorporado un nuevo atributo con la valoración de la irregularidad correspondiente a cada uno de los aforos.

\section{Resultados y discusión}

\subsection{Determinación de la irregularidad: Valores de coeficiente de caudal y desviación típica}

Desde que Masachs (1948) definiera y utilizara el coeficiente de irregularidad para analizar la irregularidad interanual de los ríos de la Península Ibérica, son numerosos los estudios hidrológicos en los que dicho coeficiente se ha empleado con ese mismo fin. Sin duda, a pesar de las limitaciones que se le reconocen, el coeficiente de irregularidad ha servido para comparar el grado de variabilidad que diferentes cursos fluviales mostraban y para comprobar su relación con la cadencia de las precipitaciones. La principal de estas limitaciones consiste en que un dato de aportación anual especialmente elevado $o$ bajo en una serie hace variar sustancialmente el valor de la irregularidad.

Masachs (1948) asignó un valor de coeficiente de irregularidad para todos los cursos fluviales peninsulares de los que existían registros instrumentales, si bien el mismo autor señaló que las series eran todavía demasiado cortas. Los valores obtenidos, como no podía ser de otro modo, fueron muy dispares, desde la regularidad de ríos de la cornisa cantábrica o cabeceras ubicadas en Pirineos, con valores de coeficiente de 2 y 3 , hasta los coeficientes por encima de 100 de diversos ríos del ámbito mediterráneo o incluso la cuenca del Guadiana. Algunos de estos valores se han confirmado en trabajos más recientes. Así por ejemplo, la muy elevada irregularidad de diversas cuencas mediterránea como las del Mijares, Segura ó Guadalentín (Gil Olcina, 1968; Conesa, 1985; Conesa y Martínez-Alcocer, 1995, Conesa y Pérez, 2014) o como las de numerosas ramblas (Triviño y Ortíz, 2004). Martín (2010) y la Agencia Catalana del Agua (2015) establecen coeficientes de irregularidad para los ríos del sector nororiental de la Península Ibérica que oscilan desde valores bajos en el Cinca o Segre, hasta valores por encima de 20 en Tordera o Francolí.

En el Plan Medioambiental del Ebro (Iberinsa, 2005) se consignan valores de coeficientes que hablan de la regularidad de ríos como el Ara o el Esera, el incremento de irregularidad en Alcanadre o Flumen y la elevada irregularidad del Guatizalema. Precisamente en el sector central del Pirineo Aragonés, García Ruiz et al (1985 y 2001) han analizado en profundidad el comportamiento de los ríos, 
estudiando entre otras características su irregularidad interanual. Señalan un incremento de la irregularidad de oeste a este, a medida que disminuye la influencia atlántica. Apuntan la mayor irregularidad de los ríos prepirenaicos, frente a la significativa regularidad del Ésera, cabecera del Cinca, Cinqueta y Ara, cursos fluviales que surcan el Alto Pirineo. Estos autores ponen en evidencia la que hemos definido como principal limitación del coeficiente de irregularidad, ya que explican cómo cambian los valores entre los dos trabajos publicados, al ampliarse el número de años con que se cuenta para el más reciente.

El coeficiente de irregularidad solo indica la relación entre el año con aportaciones más elevadas y el año con aportaciones más bajas, pero no consigue expresar si el resto de los años de la serie la aportación está muy alejada del módulo anual o poco, es decir, el grado de dispersión de las diferentes aportaciones anuales. Por ello, algunos autores han preferido utilizar para definir la irregularidad interanual el coeficiente de variación, que es la relación entre la desviación típica de una muestra y su media.

$$
C \cdot V \cdot=\frac{\sigma}{\bar{x}}
$$

Así lo hacen los mencionados García Ruiz et al. (2001) en su trabajo sobre los ríos del Pirineo aragonés y Belmar et al. (2011) en los cursos fluviales de la cuenca del Segura. También otros autores en lugares muy alejados de nuestra zona de estudio, como Clausen y Biggs (2000) en cursos de caudal continuo de Nueva Zelanda y Dettinger y Díaz (2000) que consideran 858 puntos de la red de todo el Planeta, en los que existan registros de más de 10 años.

Nosotros pensamos que la mejor forma de definir la irregularidad interanual es partiendo de los valores máximos que ésta pueda alcanzar, es decir determinando el coeficiente de irregularidad, pero también la dispersión que dentro de la serie de datos de un aforo se alcance considerando los valores de aportación de cada año hidrológico y el módulo anual. Esta dispersión puede expresarse a través de la desviación típica o también a través del coeficiente de variación.

Por ello, se ha definido la irregularidad interanual combinando los valores de coeficiente de irregularidad de cada una de las 119 estaciones de aforo consideradas con los de desviación típica, aunque hubiera sido exactamente igual utilizar los del coeficiente de variación. Con esto se consigue paliar el inconveniente que muestra el coeficiente de irregularidad y que de alguna forma le hace incompleto para indicar la irregularidad interanual de los ríos.

Los valores de coeficiente de irregularidad obtenidos son tremendamente variables (Fig. 2). Oscilan entre los 2,72 del río Irati en Arive ó los 2,78 del Noguera Ribagorzana en Pont de Suert hasta los más de 70 del río Figuerales en Valderrobres, el Gállego en Ardisa ó el Pena en Beceite. Casi fuera de rango quedan los 143,5 del río Salado en Alloz y los 163,6 del Alhama en Cintruénigo. En una parte considerable de los aforos estudiados, más del $75 \%$, el coeficiente de irregularidad se sitúa entre 3 
y 20 . Casi el $60 \%$ de los aforos analizados tienen coeficiente por debajo de 12 y el $50 \%$ inferior a 8. Esta irregularidad de las aportaciones anuales tiene una enorme relación con el ritmo y la propia irregularidad de las precipitaciones anuales (González-Hidalgo et al., 2010; Cortesi et al., 2014; Serrano-Notivoli et al., 2017). Esta correspondencia es muy directa en los tramos altos de las cuencas y se puede ir desdibujando en aquellos ríos que reciben una mayor cantidad de afluentes de entidad. Se muestra en los tres casos de la figura 3.

Figura 2. Valores de coeficiente de irregularidad y de desviación típica obtenidos para las diferentes estaciones de aforo consideradas.

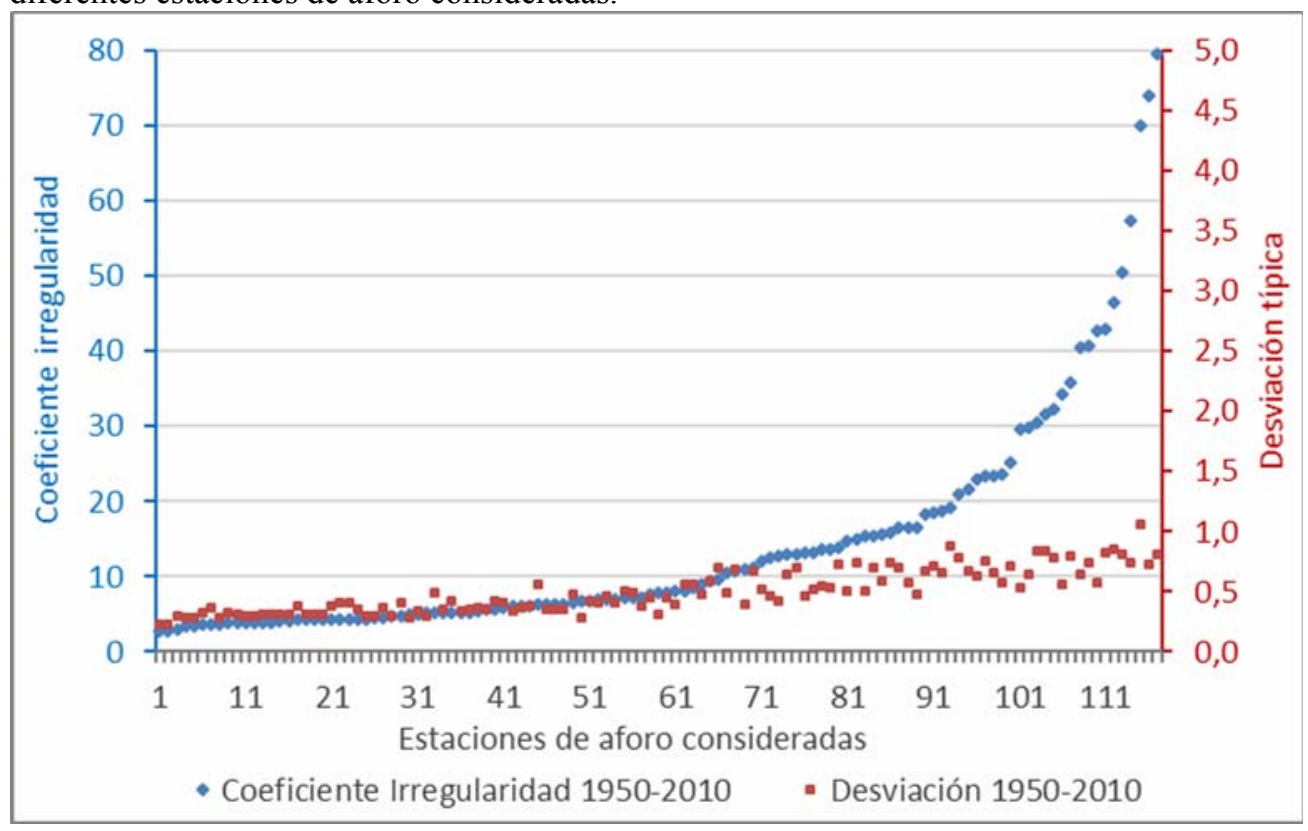

Fuente: Anuario de Aforos (MAGRAMA). Elaboración propia.

En ellos se ha considerado el módulo anual de precipitaciones y aportaciones como valor 1 y el dato de cada año se ha establecido como la razón con ese valor modular. Con la figura se intenta mostrar exclusivamente el elevado número de años en los que hay correspondencia entre los valores de las precipitaciones y las aportaciones, coincidiendo su posición en la gráfica por encima o por debajo del módulo. Así, en Boltaña hay coincidencia en el $83 \%$ de los años hidrológicos estudiados en el carácter elevado o bajo de precipitaciones y aportaciones. En Alcorisa en el 76\%. Respecto a la dispersión de los registros de aportaciones anuales en los 119 aforos analizados el resultado muestra valores de desviación típica por debajo de $0,33(33 \%)$ en el $21 \%$ de los casos, por debajo de 0,50 en un $57 \%$ de las estaciones y un $70 \%$ por debajo de 0,6 . Sólo los datos de 1 de cada 5 aforos presentan una desviación típica superior a 0,7 (Fig. 2). 
Figura 3. Relaciones entre aportaciones y precipitaciones anuales: Boltaña, Guadalope en Santolea CE y Alcorisa, Zaragoza.
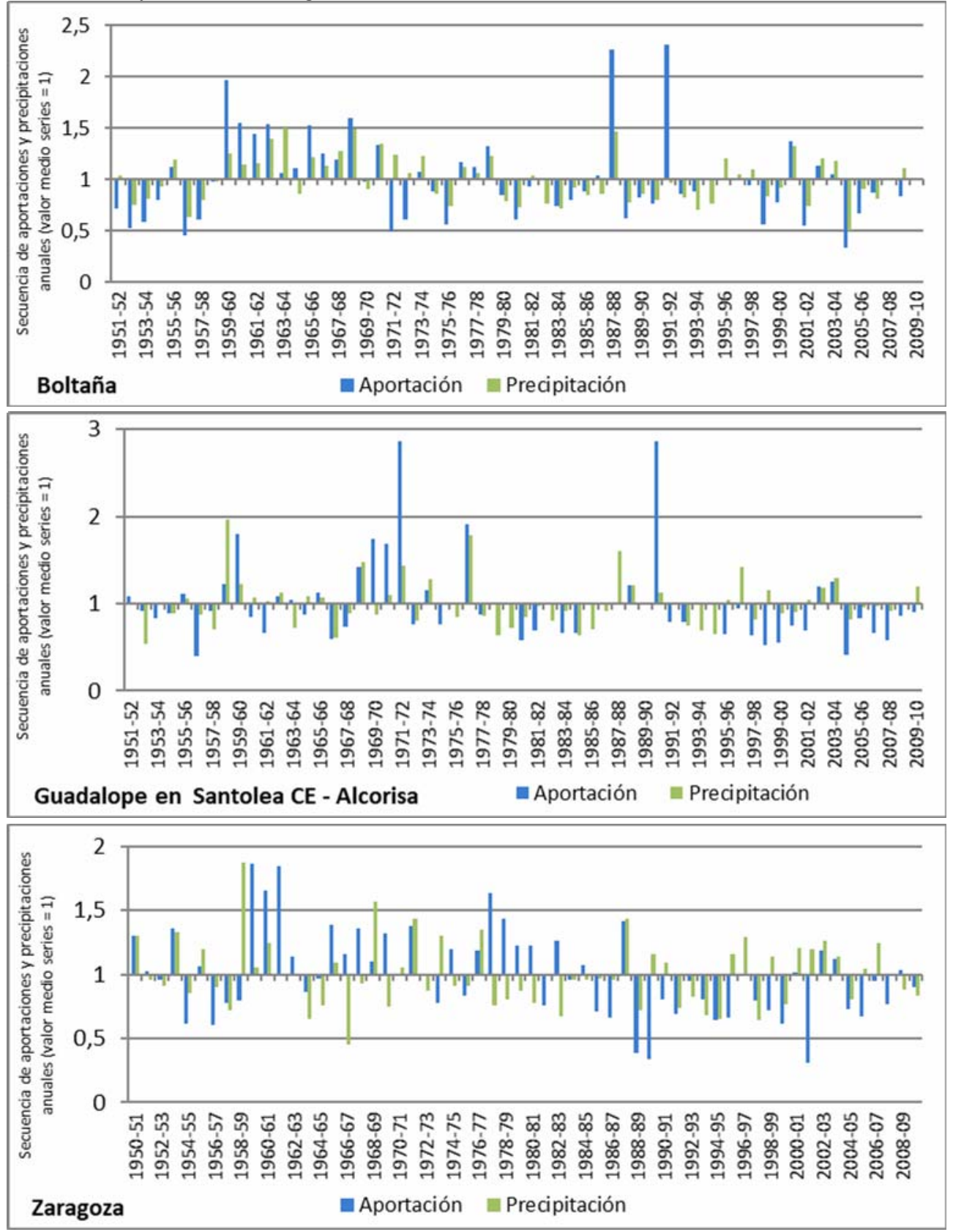

Fuentes: Anuario de Aforos y Atlas Climático de Aragón. Elaboración propia. 
Los valores de coeficiente de irregularidad y de desviación típica obtenidos para cada aforo tienen un elevado nivel de correlación, de modo que aquellos valores más bajos de coeficiente de irregularidad coinciden con una menor desviación típica, expresando por tanto un comportamiento regular de las aportaciones anuales. A medida que se van obteniendo registros más altos del coeficiente va subiendo también la dispersión de los registros anuales, con lo cual se va elevando la irregularidad interanual. En esta línea, los mayores coeficientes se relacionan con las mayores dispersiones, expresando conjuntamente fuerte irregularidad (Fig.4).

Figura 4. Correlación entre coeficiente de irregularidad y desviación típica.

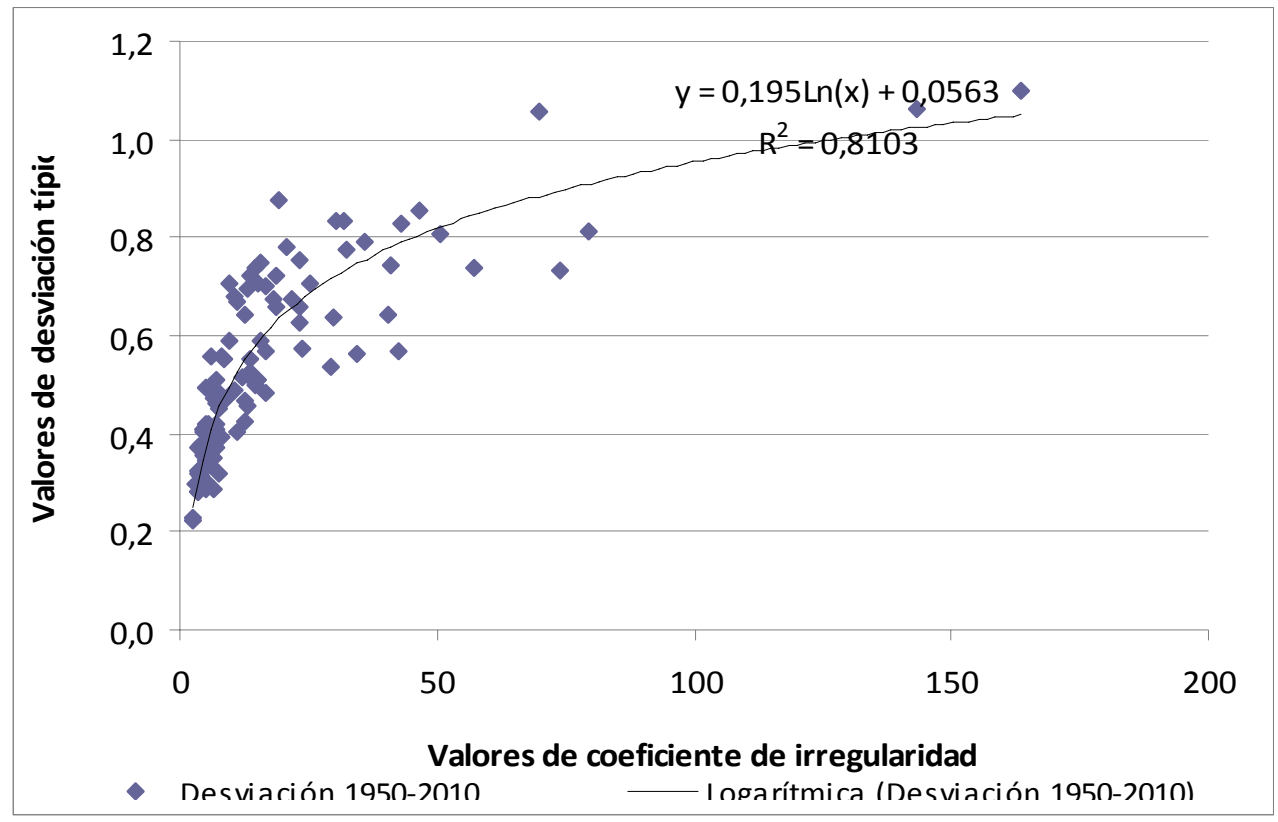

Fuente: Elaboración propia.

Esta alta correlación $(0,74)$ y el fuerte paralelismo de coeficiente de irregularidad y desviación estándar refuerza nuestra idea de que conjuntamente son una magnífica expresión de la irregularidad interanual. Como ya hemos indicado, irregularidad no sólo referida a registros extremos de una serie, sino a la dispersión de los datos en la misma.

\subsection{Propuesta de clasificación de la irregularidad interanual}

Una vez constatada la bondad del mecanismo de determinación de la irregularidad interanual, es el momento de intentar categorizar esta irregularidad y establecer diferentes tipos o niveles de ella. La definición de estos tipos, sustentada en los 
argumentos de coeficiente de irregularidad y desviación típica, ayudará a clasificar los aforos de ámbitos territoriales diversos y cursos fluviales con aportaciones y regímenes diferentes, bajo un criterio uniforme. Además, la asignación a los diferentes grupos podrá facilitar la toma de decisiones en cuanto a la manera más adecuada de gestionar recursos en las cuencas.

A partir de los datos de coeficiente de irregularidad y de desviación típica obtenidos para las 119 estaciones de aforo consideradas se ha procedido a efectuar su clasificación o tipificación. Se han tenido muy presentes las calificaciones cualitativas que autores como Masachs (1948), Dettinger y Díaz (2000), García Ruiz et al (1985 y 2001), Conesa (1985), Triviño y Ortiz (2004) y Conesa y Pérez (2014) han asociado a valores numéricos, del coeficiente de irregularidad o del coeficiente de variación, obtenidos para los cursos fluviales de sus áreas de trabajo. Con todo ello se han planteado varias opciones de clasificación, todas ellas con un número similar de categorías, pero cada una jugando con variaciones en el umbral de separación de estas categorías. Se trataba de establecer tipos bien diferenciados de los demás y con rasgos comunes bien definidos en los componentes de cada uno de ellos.

La clasificación resultante se compone de 5 niveles de irregularidad o categorías, alguno de los cuales se desdobla en dos subtipos. Cada una de estas categorías se define por un intervalo de valores del coeficiente de irregularidad y por un umbral de desviación típica (Tabla 1).

Tabla 1. Tipos o niveles de irregularidad interanual.

\begin{tabular}{|c|c|c|c|}
\hline $\begin{array}{c}\text { Tipo o nivel de irregularidad } \\
\text { interanual }\end{array}$ & Subtipos & $\begin{array}{c}\text { Coeficiente de } \\
\text { irregularidad }\end{array}$ & $\begin{array}{c}\text { Desviación } \\
\text { típica }\end{array}$ \\
\hline Regular & & $<4,00$ & $<0,33$ \\
\hline \multirow{2}{*}{ Irregularidad baja } & & $4,01-6,00$ & $<0,45$ \\
\hline \multirow{3}{*}{ Irregularidad intermedia } & $\begin{array}{c}\text { Irregularidad } \\
\text { intermedia }\end{array}$ & $6,01-12,00$ & $<0,45$ \\
\cline { 2 - 4 } & $\begin{array}{c}\text { Irregularidad } \\
\text { intermedia + }\end{array}$ & $6,01-12,00$ & $>0,45$ \\
\hline \multirow{2}{*}{ Irregularidad alta } & Irregularidad alta & $12,01-20,00$ & $0,4-0,6$ \\
\cline { 2 - 4 } & $\begin{array}{c}\text { Irregularidad alta } \\
+\end{array}$ & $12,01-20,00$ & $>0,6$ \\
\hline \multirow{3}{*}{ Irregularidad máxima } & $\begin{array}{c}\text { Irregularidad } \\
\text { máxima }\end{array}$ & $>20,01$ & $0,6-0,7$ \\
\cline { 2 - 4 } & $\begin{array}{c}\text { Irregularidad } \\
\text { máxima + }\end{array}$ & $>20,01$ & $>0,7$ \\
\hline
\end{tabular}

Fuente: Elaboración propio. 
Figura 5. Variabilidad de las aportaciones anuales en los diferentes tipos de irregularidad ${ }^{2}$.

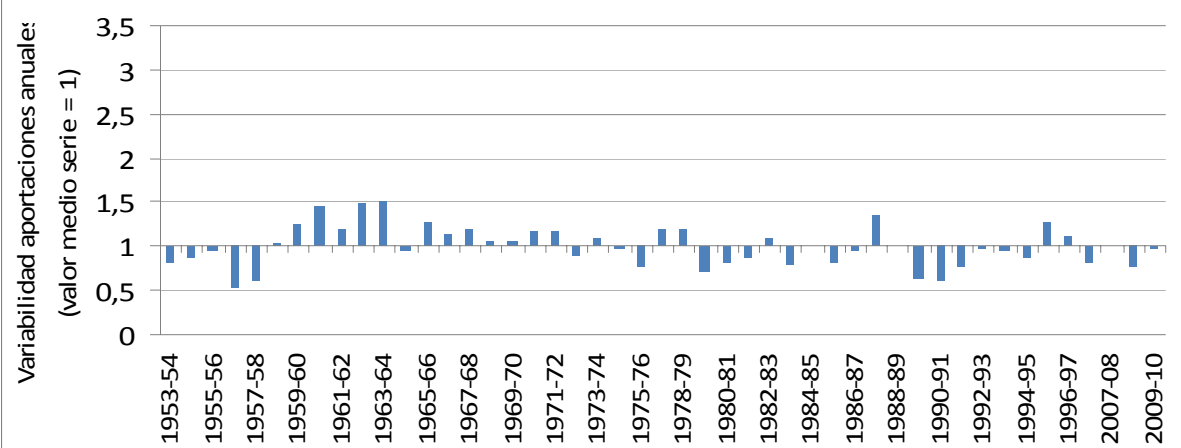

5A. NOGUERA RIBAgORZANA en Pont de Suert
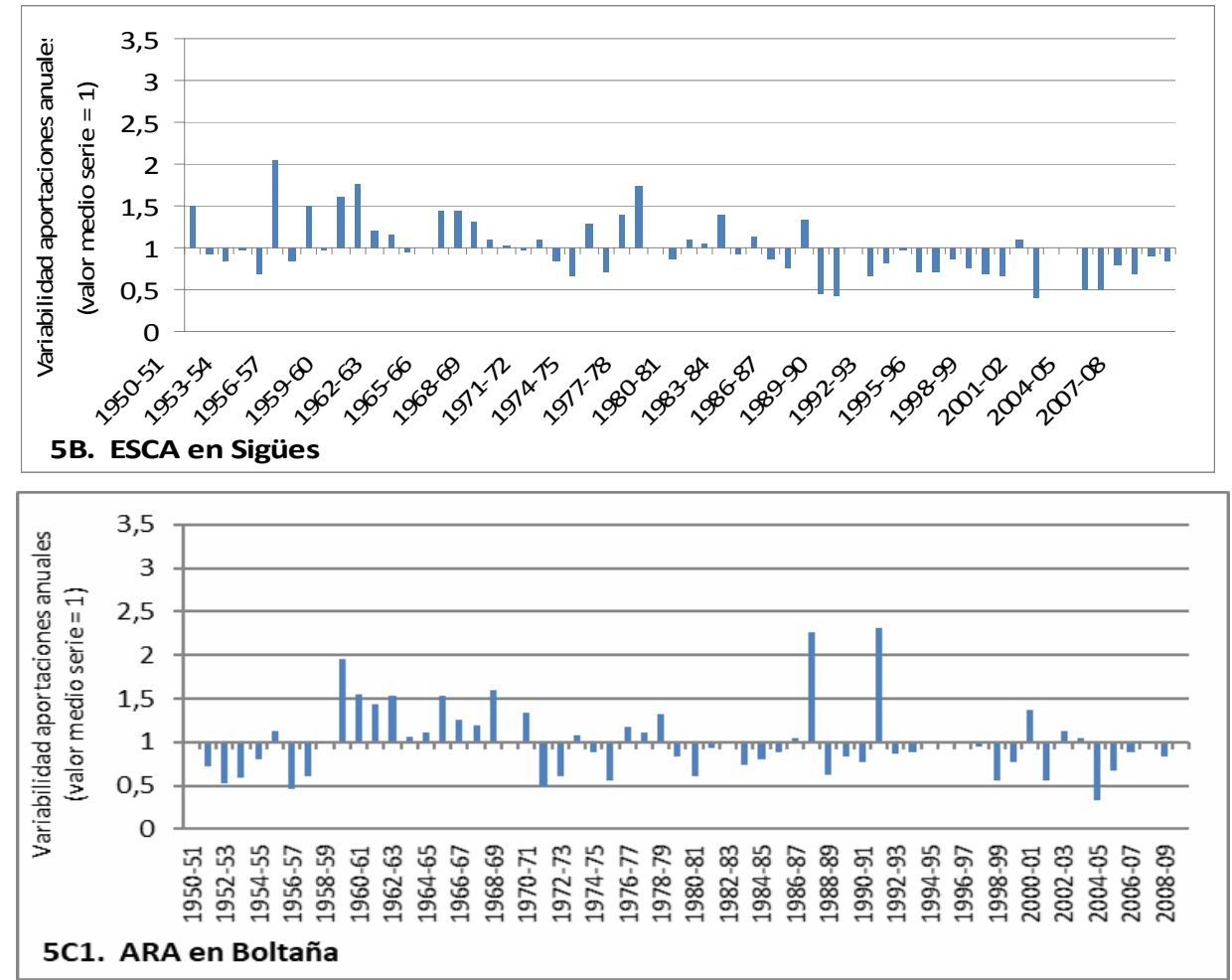

Fuente: Anuario de Aforos (MAGRAMA). Elaboración propia.

$\left.{ }^{2} 5 \mathrm{~A}\right)$ regular, 5B) irregularidad baja, 5C1) irregularidad intermedia, 5C2) irregularidad intermedia + , 5D1) irregularidad alta, 5D2) irregularidad alta,$+ 5 \mathrm{E}$ ) irregularidad máxima. 
Figura 5. Variabilidad de las aportaciones anuales en los diferentes tipos de irregularidad (continuación).
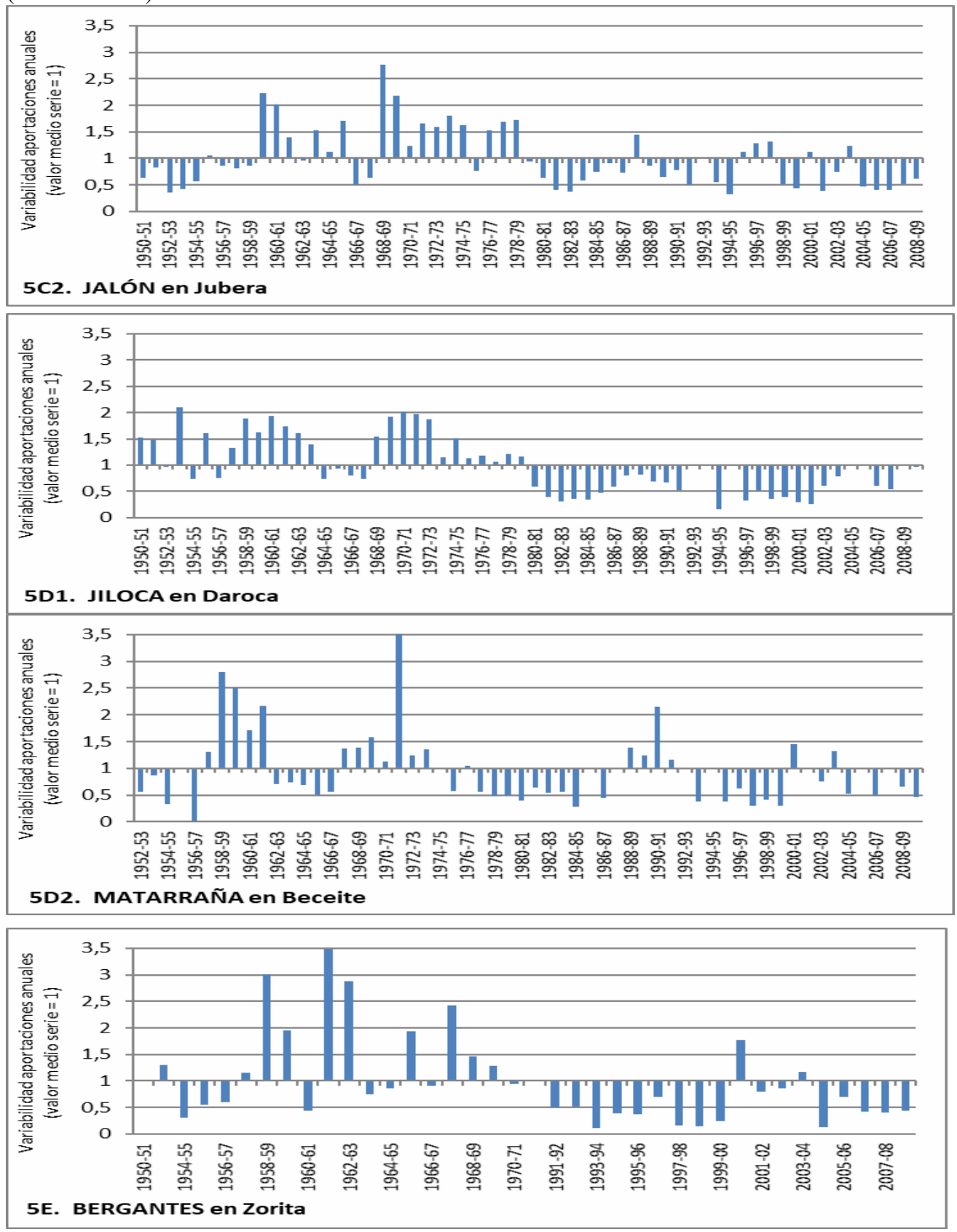

Fuente: Anuario de Aforos (MAGRAMA). Elaboración propia. 
Cursos fluviales regulares serán aquellos cuyo coeficiente de irregularidad no supere el valor 4. Es decir, el año con mayor volumen de aportaciones de la serie multiplica como mucho en cuatro veces el año con menores aportaciones. Además, la desviación típica queda por debajo de 0,33 . Se han elaborado unas gráficas (Fig. 5) que ayudan a visualizar las diferencias entre los distintos tipos de irregularidad. Los ríos regulares están representados por el aforo de Pont de Suert del río Noguera Ribagorzana (Fig. 5A), apreciándose que las aportaciones están todos los años muy próximas al valor 1 , no llegando en ningún caso a superar el $50 \%$ de ese valor ni a quedarse por debajo de la mitad. La gráfica es por tanto muy expresiva de esta regularidad que define el tipo.

La irregularidad baja se define por un coeficiente de irregularidad con valores de 4 a 6 y una desviación típica por debajo de 0,45. Es representativo el aforo del río Esca en Sigües (Fig. 5B). En él se aprecia que la desviación de las aportaciones sigue siendo relativamente baja, pero a diferencia del tipo anterior ahora sí que en algunos años se alcanzan volúmenes de aportaciones inferiores a la mitad del módulo o que lo superan en un $50 \%$.

Los ríos con irregularidad intermedia tienen coeficiente de irregularidad por encima de 6 y hasta 12. Su desviación típica puede ser variable, diferenciándose dos subtipos en función de que está se sitúe por debajo o por encima de 0,45 . En estos ríos las fluctuaciones de las aportaciones anuales muestran con frecuencia registros un $50 \%$ por encima del módulo, incluso ocasionalmente valores que duplican el módulo. En el otro sentido, son muchos los años cuyas aportaciones se quedan cerca o por debajo de la mitad de dicho módulo. Este tipo de irregularidad está representada por los casos del río Ara en Boltaña (Fig. 5C1) y del río Jalón en Jubera (Fig. 5C2). La diferenciación de los dos subtipos se basa en que en los ríos con irregularidad media + son más numerosos los años en los que las aportaciones duplican el módulo o están por encima del $50 \%$ del mismo; también, con mayor frecuencia las aportaciones quedan por debajo de la mitad del módulo.

La irregularidad alta se corresponde con coeficiente de irregularidad superior a 12 hasta 20. De nuevo la variabilidad de la dispersión de las aportaciones anuales de la serie lleva a la diferenciación de dos subtipos. Cursos o aforos de irregularidad alta son aquellos en los que la desviación típica queda entre 0,4 y 0,6 . El ejemplo del río Jiloca en Daroca (Fig. 5D1) nos muestra como en este subtipo muchos años las aportaciones prácticamente duplican el módulo y, al mismo tiempo, también muy frecuentemente los volúmenes anuales de agua no llegan a la mitad del módulo. Lo menos habitual es que las aportaciones anuales estén próximas a dicho módulo. Cursos de irregularidad alta + tienen desviaciones típicas superiores a 0,6. Presentan una acentuación de la alta variabilidad mostrada en el subtipo anterior, de modo que ya no sólo es frecuente que las aportaciones anuales dupliquen el módulo sino que hay años en que este se multiplica por 2,5 ó más. Del mismo modo, con gran frecuencia las aportaciones quedan reducidas a la mitad del módulo e incluso se pueden reducir hasta cerca de una cuarta parte, tal como se aprecia en el caso del río Matarraña en Beceite (Fig. 5D2). 
La irregularidad máxima es la alcanzada cuando el coeficiente de irregularidad supera el valor 20. Se distinguen dos subtipos en función de que la desviación típica, siempre elevada, quede ente 0,6 y 0,7 (irregularidad máxima) o supere el 0,7 (irregularidad máxima + ). Se ejemplifica con el río Bergantes en Zorita (Fig. 5E), donde se aprecia la enorme variabilidad de las aportaciones en distintos años: algunos próximos al módulo; otros alcanzando el $200 \%$, 250\% e incluso más del $300 \%$ de dicho módulo; finalmente, un considerable porcentaje de años en que las aportaciones son inferiores al $50 \%$ del módulo y bastantes en las que incluso están por debajo del $25 \%$ de ese módulo.

Es conveniente hacer alusión a un hecho que se aprecia en las diferentes gráficas que integran la figura 5: en todas ellas se constata, aunque en diferente grado, que a partir de la década de los 80 del siglo XX hay un gran predominio de los años cuya disponibilidad de caudal está por debajo del módulo. Este hecho, que ya ha sido señalado por diferentes autores en la cuenca del Ebro (García Ruiz et al., 2001; Frutos et al., 2004; Cuadrat et al., 2007, García-Ruiz et al., 2011; Lorenzo-Lacruz et al., 2012; Sánchez-Fabre et al., 2013), se asocia tanto a un descenso de las precipitaciones anuales como, en mayor medida, a cambios en los usos del suelo muy ligados al abandono de campos de cultivo y zonas de pastoreo. Estos cambios de uso han determinado un evidente incremento de la cubierta vegetal, con la consiguiente variación en la distribución del agua entre los diferentes procesos del ciclo hidrológico, que finalmente supone un descenso en el aporte de agua que llega hasta los ríos.

\subsection{La irregularidad interanual en la cuenca del Ebro}

Uno de los objetivos de este trabajo es la determinación del nivel de irregularidad que presentan los ríos de la cuenca del Ebro en diferentes estaciones de aforo. Esto debe servir, al mismo tiempo, para comprobar la bondad de esta propuesta de clasificación de la irregularidad interanual de las aportaciones fluviales. Los 119 aforos seleccionados se distribuyen por todo el territorio de la cuenca de una forma compensada, dejando bien representados los diferentes sectores de la misma.

Al proceder a la clasificación de la irregularidad interanual de las aportaciones, aplicando los criterios fijados en el apartado anterior, lo primero que se observa es que todos los grupos están bien representados. Incluso los cinco grandes tipos en porcentajes bastante similares, con la excepción del correspondiente a cursos fluviales regulares, que agrupa un menor porcentaje de casos. Este hecho no nos debe extrañar considerando el carácter mediterráneo que la cuenca del Ebro posee en gran parte de su extensión. Los porcentajes de aforos incluidos en cada uno de los cinco grandes tipos se muestran en la figura 6. Destaca por ser ligeramente más elevado el número de aforos encuadrados en el tipo de irregularidad intermedia. En el otro extremo, el menor número de aforos $\mathrm{y}$, por tanto, tramos fluviales que pueden calificarse de regulares. 
Figura 6. Reparto porcentual de los aforos analizados en los tipos de irregularidad interanual.

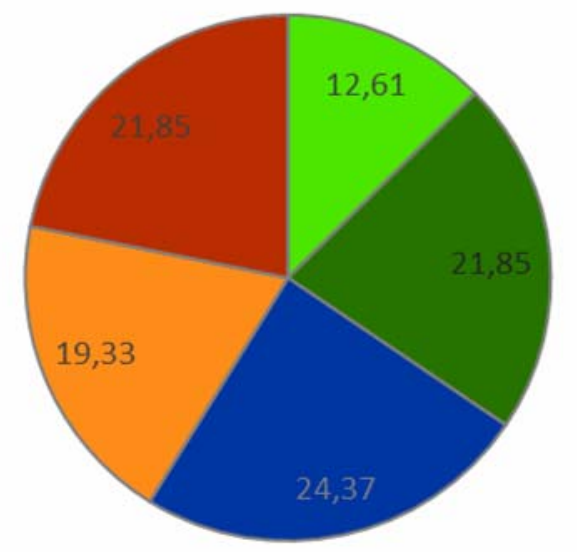

Regular

Irregularidad baja

E Irregularidad intermedia

Irregularidad alta

Irregularidad máxima

Fuente: Elaboración propia.

La distribución de la irregularidad interanual de las aportaciones aparece recogida en el mapa de la figura 7. Antes de hacer referencia a los rasgos principales de esa ordenación es preciso indicar que se han representado con símbolos circulares aquellos aforos donde la serie se inicia a mitad del siglo $\mathrm{XX}$ y triangulares los correspondientes a series iniciadas en el último cuarto de ese siglo. Los primeros cuentan en su mayor parte con más de 50 años con registros de aportación anual, en tanto que los segundos cuentan con más de 30 años con dato.

El primer rasgo relevante de la distribución de los diferentes tipos de irregularidad interanual en la cuenca del Ebro es el enorme contraste entre la margen derecha y la margen izquierda. No puede extrañar, dadas las diferencias que entre las subcuencas de estas dos márgenes del Ebro existen, tanto desde el punto de vista topográfico, como litológico, biogeográfico y climático. Estas diferencias son las causantes de grandes variaciones en los caudales y también en el reparto estacional de los mismos, por lo que también cabría esperarlas en la irregularidad interanual. Es en la margen izquierda donde se concentran casi todos los aforos con valores de coeficiente de caudal y desviación típica que los definen como regulares o de irregularidad baja. Por el contrario, en la margen derecha hay un predominio de aforos donde se constata una irregularidad alta o máxima. 
Figura 7. Categorías de irregularidad interanual en la cuenca del Ebro.

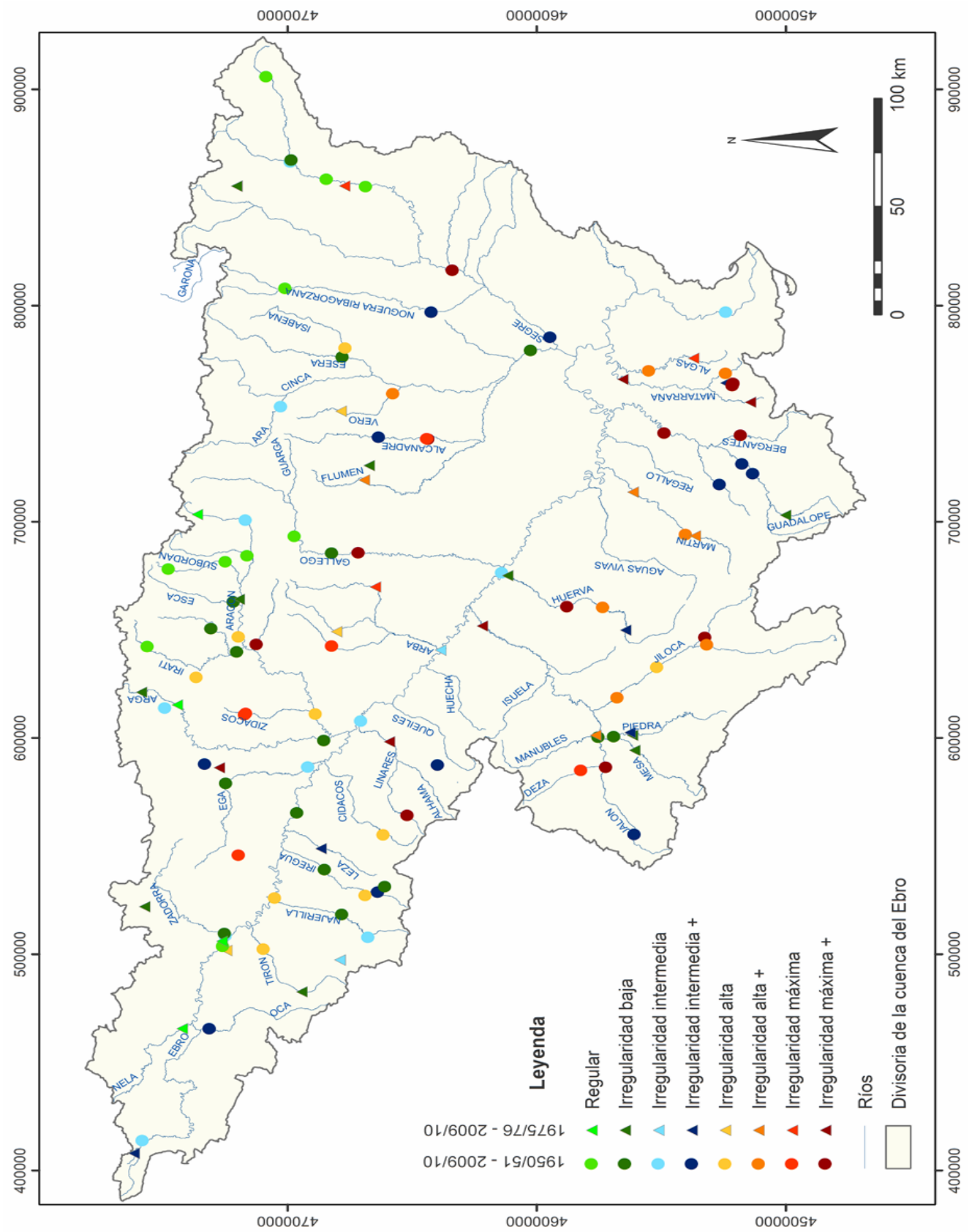

Fuentes: Anuario de Aforos (MAGRAMA) y SIT-Ebro. Elaboración propia. 
En la margen izquierda se pueden distinguir tres situaciones dominantes:

- Las cabeceras de los ríos pirenaicos muestran una gran regularidad en la disponibilidad de agua en diferentes años o, como mucho, una irregularidad baja. Esto se aprecia muy claramente en los afluentes de la margen derecha del río Aragón, en el río Ésera y en el Segre.

- Los tramos medios de los ríos, que atraviesan las sierras prepirenaicas, o los cursos fluviales que nacen en esta unidad morfoestructural prepirenaica, perteneciente a la cadena montañosa de los Pirineos, son los únicos en esta margen izquierda con irregularidad alta e incluso máxima.

- Las cuencas bajas de los ríos nacidos en las cumbres pirenaicas adoptan un nivel de irregularidad bajo o intermedio. Así está muy bien definido en los ríos Zadorra, Ega y Cinca-Segre.

En la margen derecha cualquier situación distinta a una irregularidad alta o máxima aparece como excepcional. Sólo en el sector más occidental de esta margen derecha dominan los aforos y ríos con irregularidad baja o media (Najerilla, Iregua, Leza), sin duda como consecuencia del influjo regulador del Cantábrico y los frentes que desde él penetran en la Península. La cuenca del Leza parece marcar el límite del dominio de esa influencia sobre la mediterránea y por tanto del paso a ese comentado protagonismo de la irregularidad alta o máxima en el funcionamiento de los ríos.

Entre esos aforos que constatan tan fuerte irregularidad, llama la atención la puntual presencia de aforos con irregularidad intermedia (Guadalope-Guadalopillo, cabecera del Jalón) o incluso baja (río Pitarque, ríos Mesa y Piedra). No cabe duda de que el componente litológico, ya que son cuencas con casi exclusiva presencia y afloramiento de formaciones carbonatadas, es el causante directo de esta baja irregularidad, e incluso también puede intervenir en los casos de irregularidad media.

Finalmente, el Ebro, río principal de la cuenca, presenta en casi todo su recorrido una irregularidad intermedia que ya había sido definida como tal por Frutos et al. (2004).

\section{Conclusiones}

La irregularidad interanual es una de las principales propiedades del comportamiento hidrológico de los ríos, a la que se ha dedicado menos atención que al régimen estacional de los caudales o a las crecidas.

Tradicionalmente, desde Masachs (1948), la irregularidad interanual se ha definido mayoritariamente a partir del coeficiente de caudal. Aunque válido, este sistema puede llevar a valores muy sesgados tanto por el uso de series de datos de caudales o aportaciones poco prolongadas en el tiempo como por la presencia de un valor muy extremo tanto en un año con una aportación excepcionalmente elevada como escasa. El uso de la desviación típica para analizar la dispersión de los datos anuales respecto al módulo de la serie, sin duda, corrige ese sesgo. Por ello, se ha establecido como 
criterio para definir la irregularidad interanual de un aforo la combinación del valor de coeficiente de caudal con el valor de la desviación típica de la serie de datos. Para homogeneizar los resultados es conveniente que la serie temporal sea similar en los aforos analizados y se ha optado por llevar los valores de aportaciones a una razón de números en la que el módulo sea el valor 1 y los demás registros anuales estén referidos a él.

Calculados los valores de coeficiente de irregularidad y desviación típica para 119 aforos de la cuenca del Ebro, se aprecia una tremenda irregularidad en los primeros (desde 2,72 a 143,5 ) y respecto a la desviación típica: en un $21 \%$ de los casos resulta inferior a 0,33 , en un $57 \%$ está por debajo de 0,5 , en el $70 \%$ de los casos por debajo de 0,6 y algo menos del $20 \%$ supera el 0,7 .

A partir de la consideración conjunta de coeficiente de irregularidad y desviación típica, tras comprobar la elevada correlación entre ambos, y teniendo en cuenta los resultados obtenidos para los 119 aforos analizados en la cuenca del Ebro se han definido 5 tipos o niveles de irregularidad interanual: regular, irregularidad baja, irregularidad intermedia, irregularidad alta e irregularidad máxima. Dentro de las tres últimas categorías se han diferenciado dos subtipos en función del grado de dispersión de los datos de la serie (desviación típica). Cada una de estas categorías de irregularidad posee un umbral claro con las contiguas, que se aprecia no sólo en los valores numéricos sino al analizar gráficamente la dispersión de los datos de las aportaciones anuales en aforos expresivos de cada uno de los tipos.

Asignada categoría de irregularidad a cada uno de los aforos considerados, su cartografía permite ver la distribución de la irregularidad interanual en la cuenca del Ebro. Destaca como aspecto más relevante el enorme contraste entre las márgenes izquierda y derecha. Los ríos de la margen izquierda son regulares en las cabeceras pirenaicas, algo menos en sus tramos bajos y, excepcionalmente, irregulares aquellos cursos fluviales o tramos instalados en las sierras prepirenaicas. Los de la margen derecha del Ebro son ríos de irregularidad alta o máxima, con las únicas excepciones de los situados más al oeste (Najerilla, Iregua, Leza), ya que su irregularidad se ve mitigada por la acción del Cantábrico, o de aquellos cuyas cuencas están asentadas sobre formaciones carbonatadas que ejercen una clara acción regularizadora. El Ebro, río principal de la cuenca, presenta en casi todo su recorrido una irregularidad intermedia.

En definitiva, la propuesta de evaluación de la irregularidad interanual parece adecuada ya que su aplicación en la cuenca del Ebro muestra unos resultados acordes a los datos aportados por diferentes estudios que anteriormente evaluaron por separado coeficiente de caudal o coeficiente de variación y también coherentes con las características naturales (topografía, precipitaciones, vegetación, litología...) de los diferentes sectores de la cuenca del Ebro. 


\section{Bibliografía}

Agencia Catalana del Agua (2015): Anexo III, Inventario de recursos hídricos totales. En Agencia Catalana del Agua: Plan de gestión del distrito de cuenca fluvial de Cataluña 2016-2021. Barcelona. Generalitat de Catalunya.

Belmar, O., Velasco, J. y Martínez-Capel, F. (2011): Hydrological Classification of Natural Flow Regimes to Support Environmental Flow Assessments in Intensively Regulated Mediterranean Rivers, Segura River Basin (Spain). Environmental Management, 47, 9921004. DOI 10.1007/s00267-011-9661-0.

Clausen, B. y Biggs, B.J.F. (2000): Flow variables for ecological studies in temperate streams: groupings based on covariance. Journal of Hydrology, 237, 184-197.

Conesa, C. (1985): Inundaciones en Lorca (Murcia): Riesgo y expectación. Papeles de Geografía (Física), 10, 33-47.

Conesa, C. y Martínez-Alcocer, P. (1995): Magnitud y frecuencia de sucesos hidromorfológicos del bajo Segura anteriores a su encauzamiento. Papeles de Geografía, 22, 67-86.

Conesa, C. y Pérez, P. (2014): Alteraciones geomorfológicas recientes en los sistemas fluviales mediterráneos de la Península Ibérica. Síntomas y problemas de incisión en los cauces. Revista de Geografía Norte Grande, 59, 25-44.

Cortesi, N., González-Hidalgo, J.C., Brunetti, M. y De Luis, M. (2014): Spatial variability of precipitation in Spain. Regional Environmental Change, 14, 1743-1749. DOI: 10.1007/s10113-012-0402-6.

Cuadrat, J.M., Saz, M.A., Vicente, S.M. y González-Hidalgo, J.C. (2007): Water resources and precipitation trends in Aragón. Water Resources Development, 23, 107-123. DOI: 10.1080/07900620701189400.

Davy, L. (1975): L'Ebre, etude hydrologique. Thése d'Etat. Université de Lille III.

Dettinger, M.D. y Díaz, H.F. (2000): Global Characteristics of Stream Flow Seasonality and Variability. Journal of Hydrometeorology, vol. 1, 289-310.

Frutos, L.M., Ollero, A. y Sánchez Fabre, M. (2004): Caracterización del Ebro y su cuenca y variaciones en su comportamiento hidrológico. En Gil Olcina, A. (Dir.): Alteración de los regímenes fluviales peninsulares (1901-2000). Instituto Euromediterráneo de Hidrotécnia Cajamurcia, 233-280.

García Ruiz, J.M., Beguería, S., López, J.I., Lorente, A. y Seeger, M. (2001): Los recursos hídricos superficiales del Pirineo Aragonés y su evolución reciente. Logroño. Geoforma ediciones.

García-Ruiz, J.M., López-Moreno, J.I., Serrano-Vicente, S.M., Beguería, S. y Lasanta, T. (2011): Mediterranean wáter resources in a global change scenario. Earth Science Rewiews, 105, 121-139. DOI: 10.1016/j.earscirev.2011.01.006.

García Ruiz, J.M., Puigdefábregas, J. y Creus, J. (1985): Los recursos hídricos superficiales del Alto Aragón. Huesca. Colección de estudios altoaragoneses, 2. Instituto de Estudios Altoaragoneses.

Gil Olcina, A. (1968): El régimen del río Guadalentín. Cuadernos de Geografía, 5, 1-19.

Gil Olcina, A. (1988): Precipitaciones y regímenes fluviales en la vertiente mediterránea española. B.A.G.E., 7, 1-12. 
Gobierno Vasco (2003): Estudio de evaluación de los recursos hídricos totales en el ámbito de la CAPV. Síntesis. Vitoria. Dirección de Aguas, Departamento de Ordenación del Territorio y Medio Ambiente, Gobierno Vasco.

González-Hidalgo, J.C., Brunetti, M. y De Luis, M. (2010): Precipitation trends in Spanish Water Planning Divisions (Confederaciones Hidrográficas) between 1946-2005. Climate Research, 3, 215-228. DOI: 10.3354/cr00937.

IBERINSA (2005): Redacción y desarrollo del Plan medioambiental del Ebro y tramo bajo del Cinca. Madrid. Departamento de Medio Ambiente, Gobierno de Aragón.

Iglesias, A., Estrela, T. y Gallart, F. (2005): Impactos sobre los recursos hídricos. En Moreno, J.M. (Dir.): Evaluación Preliminar de los Impactos en España por Efecto del Cambio Climático, Madrid, Ministerio de Medio Ambiente.

Lorenzo-Lacruz, J., Vicente-Serrano, S.M., López-Moreno, J.I., Morán-Tejeda, E. y Zabalza, J. (2012): Recent trends in Iberian stream flows (1945-2005). Journal of Hydrology, 414-415, 463-475. 10.1016/j.jhydrol.2011.11.023

Martín, J. (2010): Influencia de los patrones de variabilidad de baja frecuencia WeMO y NAO sobre el caudal de los ríos en Cataluña (1951-2000). Boletín de la Asociación de Geógrafos Españoles, 54, 237-257.

Masachs, V. (1948): El régimen de los ríos Peninsulares. Madrid. Instituto "Lucas Mallada", CSIC.

Morales, A., Rico, A.M. y Hernández, M. (2005): El trasvase Tajo-Segura. Observatorio Medioambiental, 8, 73-110.

Ollero, A. (1996): El curso medio del Ebro: geomorfología fluvial, ecogeografía y riesgos. Zaragoza. Serie Investigación, 4. Consejo de Protección de la Naturaleza de Aragón.

Restrepo, J.D. y Kjerfve, B. (2000): Magdalena river: interanual variability (1975-1995) and revised wáter discharge and sediment load estimates. Journal of Hydrology, 235, 137-149.

Robertson, A.W. y Mechoso, C.R. (1998): Interannual and Decadal Cycles in River Flows of Southeastern South America. Journal of Climate, 11, 2570-2581.

Sánchez-Fabre, M., Ollero, A., Mora, D., Del Valle, J. y Ballarín, D. (2013): Los ríos de la provincia de Teruel. Teruel, Instituto de Estudios Turolenses.

Serrano-Notivoli, R., Beguería, S., Saz, M.A., Longares, L.A., De Luis, M. (2017): SPREAD: A high-resolution daily gridded precipitation dataset for Spain - an extreme events fre quency and intensity overview. Earth System Science Data, 9, 721-738. DOI: 105194/essd-9-721-2017

Triviño, A. y Ortíz, S. (2004): Metodología para la modelación distribuida de la escorrentía superficial y la delimitación de zonas inundables en ramblas y ríos-rambla mediterráneos. Investigaciones Geográficas, 35, 67-83.

Wang, H., Yang, Z., Saito, Y., Liu, J.P. y Sun, X. (2006): Interannual and seasonal variation of the Huanghe (Yellow River) water discharge over the past 50 years: Connections to impacts from ENSO events and dams. Global and Planetary Change, 50, 212-225. DOI:10.1016/j.gloplacha.2006.01.005 


\section{Anexo I}

Estaciones de aforo de la Cuenca del Ebro consideradas

\begin{tabular}{|c|c|c|c|c|}
\hline Código & Nombre & Río & Serie 1950-2010 & Serie $1975-2010$ \\
\hline 9001 & Miranda de Ebro & EBRO & $\mathrm{X}$ & \\
\hline 9002 & Castejón & EBRO & $\mathrm{X}$ & \\
\hline 9003 & Andosilla & EGA & $\mathrm{X}$ & \\
\hline 9004 & Funes & ARGA & $\mathrm{X}$ & \\
\hline 9005 & Caparroso & ARAGÓN & $\mathrm{X}$ & \\
\hline 9006 & Marañón & EGA & $\mathrm{X}$ & \\
\hline 9007 & Cetina & JALÓN & $\mathrm{X}$ & \\
\hline 9008 & Nuévalos & PIEDRA & & $\mathrm{X}$ \\
\hline 9010 & Daroca & JILOCA & $\mathrm{X}$ & \\
\hline 9011 & Zaragoza & EBRO & $\mathrm{X}$ & \\
\hline 9012 & Ardisa & GÁLLEGO & $\mathrm{X}$ & \\
\hline 9013 & Graus & ESERA & $\mathrm{X}$ & \\
\hline 9014 & Híjar & MARTÍN & $\mathrm{X}$ & \\
\hline 9015 & Alcañiz & GUADALOPE & & $\mathrm{X}$ \\
\hline 9017 & Fraga & CINCA & $\mathrm{X}$ & \\
\hline 9018 & Jaca & ARAGÓN & $\mathrm{X}$ & \\
\hline 9021 & Puigcerdá & SEGRE & $\mathrm{X}$ & \\
\hline 9022 & Seo de Urgel & VALIRA & $\mathrm{X}$ & \\
\hline 9023 & Seo de Urgel & SEGRE & $\mathrm{X}$ & \\
\hline 9025 & Serós & SEGRE & $\mathrm{X}$ & \\
\hline 9026 & Arroyo & EBRO & $\mathrm{X}$ & \\
\hline 9027 & Tortosa & EBRO & $\mathrm{X}$ & \\
\hline 9030 & Santolea CE & GUADALOPE & $\mathrm{X}$ & \\
\hline 9031 & Zorita & BERGANTES & $\mathrm{X}$ & \\
\hline 9032 & Peralta de Alfoc. & GUATIZALEMA & $\mathrm{X}$ & \\
\hline 9033 & Peralta de Alfoc. & ALCANADRE & $\mathrm{X}$ & \\
\hline 9034 & Mansilla & NAJERILLA & $\mathrm{X}$ & \\
\hline 9035 & Villoslada & IREGUA & $\mathrm{X}$ & \\
\hline 9036 & Islallana & IREGUA & $\mathrm{X}$ & \\
\hline 9038 & Torremontalvo & NAJERILLA & $\mathrm{X}$ & \\
\hline 9039 & Ortigosa & ALBERCOS & $\mathrm{X}$ & \\
\hline 9040 & Boltaña & ARA & $\mathrm{X}$ & \\
\hline 9041 & Navarrete del R. & PANCRUDO & $\mathrm{X}$ & \\
\hline 9042 & Calamocha & JILOCA & $\mathrm{X}$ & \\
\hline 9043 & San Pedro Manrique & LINARES & $\mathrm{X}$ & \\
\hline 9044 & Yanguas & CIDACOS & $\mathrm{X}$ & \\
\hline 9046 & Lecina de Barcabo & VERO & & $\mathrm{X}$ \\
\hline 9047 & Capella & ISÁBENA & $\mathrm{X}$ & \\
\hline 9048 & Anguiano & NAJERILLA & $\mathrm{X}$ & \\
\hline 9049 & Devanos & AÑAMAZA & $\mathrm{X}$ & \\
\hline 9050 & Cuzcurrita & TIRÓN & $\mathrm{X}$ & \\
\hline 9052 & Beceite & MATARRAÑA & $\mathrm{X}$ & \\
\hline 9055 & Morata de J. & JILOCA & $\mathrm{X}$ & \\
\hline 9056 & Jaraba & MESA & & $\mathrm{X}$ \\
\hline
\end{tabular}




\begin{tabular}{|c|c|c|c|c|}
\hline 9057 & Embid de Ariza & DEZA & $\mathrm{X}$ & \\
\hline 9058 & Jubera & JALÓN & $\mathrm{X}$ & \\
\hline 9059 & Santa Eulalia & GÁLLEGO & $\mathrm{X}$ & \\
\hline 9060 & Gallur & ARBA & & $\mathrm{X}$ \\
\hline 9061 & Javierregay & SUBORDÁN & $\mathrm{X}$ & \\
\hline 9062 & Biniés & VERAL & $\mathrm{X}$ & \\
\hline 9063 & Sigües & ESCA & $\mathrm{X}$ & \\
\hline 9064 & Aspurz & SALAZAR & $\mathrm{X}$ & \\
\hline 9065 & Liédana & IRATI & $\mathrm{X}$ & \\
\hline 9066 & Arive & IRATI & $\mathrm{X}$ & \\
\hline 9067 & Olave & ULZAMA & $\mathrm{X}$ & \\
\hline 9071 & Estella & ARGA & $\mathrm{X}$ & \\
\hline 9073 & Sangüesa & ONSELLA & X & \\
\hline 9074 & Arce & ZADORRA & $\mathrm{X}$ & \\
\hline 9075 & Berantevilla & AYUDA & $\mathrm{X}$ & \\
\hline 9078 & Garinoain & CEMBORAIN & $X$ & \\
\hline 9079 & Urroz Villa & ERRO & $\mathrm{X}$ & \\
\hline 9080 & Zuriza & VERAL & $\mathrm{X}$ & \\
\hline 9083 & Oliana & SEGRE & $X$ & \\
\hline 9084 & Alloz & SALADO & & $\mathrm{X}$ \\
\hline 9086 & Barasoain & ZIDACOS & $\mathrm{X}$ & \\
\hline 9087 & Grisén & JALÓN & & $\mathrm{X}$ \\
\hline 9088 & Pitarque & FORTANETE & & $\mathrm{X}$ \\
\hline 9091 & Lascellas & ALCANADRE & $\mathrm{X}$ & \\
\hline 9092 & Trespanerde & NELA & & $\mathrm{X}$ \\
\hline 9093 & Oña & OCA & $\mathrm{X}$ & \\
\hline 9095 & Barbastro & VERO & $\mathrm{X}$ & \\
\hline 9096 & Balaguer & SEGRE & $\mathrm{X}$ & \\
\hline 9097 & La Piñana & NOG. RIBAGORZANA & $\mathrm{X}$ & \\
\hline 9100 & Berge & GUADALOPILLO & $\mathrm{X}$ & \\
\hline 9101 & Yesa PP & ARAGÓN & $\mathrm{X}$ & \\
\hline 9105 & Mezalocha & HUERVA & $\mathrm{X}$ & \\
\hline 9106 & Santolea PP & GUADALOPE & $\mathrm{X}$ & \\
\hline 9109 & Valderrobres & PEÑA & & $\mathrm{X}$ \\
\hline 9110 & Beceite & PEÑA & $\mathrm{X}$ & \\
\hline 9111 & Orgaña & SEGRE & $\mathrm{X}$ & \\
\hline 9113 & Valderrobres & FIGUERALES & $\mathrm{X}$ & \\
\hline 9118 & Oliete & MARTÍN & $\mathrm{X}$ & \\
\hline 9120 & Mendavia & EBRO & $\mathrm{X}$ & \\
\hline 9123 & Anzánigo & GÁLLEGO & $\mathrm{X}$ & \\
\hline 9124 & Las Torcas & HUERVA & $\mathrm{X}$ & \\
\hline 9125 & Carenas & PIEDRA & $\mathrm{X}$ & \\
\hline 9126 & Ateca & JALÓN & $\mathrm{X}$ & \\
\hline 9127 & Alcaine & MARTÍN & & $\mathrm{X}$ \\
\hline 9129 & Tranquera & ORTIZ & & $\mathrm{X}$ \\
\hline 9135 & Alins & TOR & & $\mathrm{X}$ \\
\hline 9137 & Pont de Suert & NOG. RIBAGORZANA & $\mathrm{X}$ & \\
\hline 9142 & Lumbreras & LUMBRERAS & $\mathrm{X}$ & \\
\hline 9148 & Coll de Nargo & SELLENT & & $\mathrm{X}$ \\
\hline 9150 & Estenoz & SALADO & $\mathrm{X}$ & \\
\hline
\end{tabular}




\begin{tabular}{|l|l|l|l|l|}
\hline 9152 & Eugui & ARGA & & $\mathrm{X}$ \\
\hline 9153 & Horta de S. Juan & ALGÁS & $\mathrm{X}$ \\
\hline 9154 & Peñarroya & TASTAVINS & $\mathrm{X}$ \\
\hline 9155 & Biota & ARBA DE LUE. & $\mathrm{X}$ \\
\hline 9157 & Azarrulla & OJA & & $\mathrm{X}$ \\
\hline 9158 & S. Miguel de P.. & TIRÓN & & $\mathrm{X}$ \\
\hline 9159 & Huarte & ARGA & $\mathrm{X}$ \\
\hline 9164 & Canfranc & ARAGÓN & $\mathrm{X}$ \\
\hline 9165 & Miranda & BAYAS & & $\mathrm{X}$ \\
\hline 9170 & Yesa CE & ARAGÓN & $\mathrm{X}$ \\
\hline 9176 & Nonaspe & MATARRAÑA & $\mathrm{X}$ \\
\hline 9177 & Batea & ALGÁS & & \\
\hline 9178 & Reinosa & EBRO & $\mathrm{X}$ \\
\hline 9184 & Ateca & MANUBLES & $\mathrm{X}$ \\
\hline 9185 & Cintruénigo & ALHAMA & $\mathrm{X}$ \\
\hline 9186 & Sádaba & ARBA DE RIGUEL & & $\mathrm{X}$ \\
\hline 9187 & Erla & ARBA DE BIEL & & $\mathrm{X}$ \\
\hline 9189 & Oron & ORONCILLO & & $\mathrm{X}$ \\
\hline 9190 & Quicena & FLUMEN & $\mathrm{X}$ \\
\hline 9192 & Siétamo & GUATIZALEMA & & $\mathrm{X}$ \\
\hline 9197 & Leza del río Leza & LEZA & & $\mathrm{X}$ \\
\hline 9198 & Alins & VALFARRERA & & \\
\hline 9215 & Cerveruela & HUERVA & & \\
\hline 9216 & Zaragoza & HUERVA & & \\
\hline 9221 & Larrinoa & SUBIALDE & & \\
\hline & & & & \\
\hline
\end{tabular}

